\title{
Control Model on Transmission Dynamic of Conjunctivitis During Harmattan in Public Schools
}

\author{
Michael Uchenna, Offia Akachukwu, Elebute Kafayat \\ Department of Mathematics, Alex Ekwueme Federal University Ndufu Alike, Ikwo, Abakaliki, Nigeria \\ Email address: \\ Michael.uchenna@funai.edu.ng(M.Uchenna),offia.akachukwu@funai.edu.ng(O.Akachukwu), elebute.kafayat@funai.edu.ng(E.Kafayat) \\ To cite this article: \\ Michael Uchenna, Offia Akachukwu, Elebute Kafayat. Control Model on Transmission Dynamic of Conjunctivitis During Harmattan in \\ Public Schools. Applied and Computational Mathematics. Vol. 8, No. 2, 2019, pp. 29-36. doi: 10.11648/j.acm.20190802.11
}

Received: March 12, 2019; Accepted: April 15, 2019; Published: May 15, 2019

\begin{abstract}
Developing countries are prone to some outburst of epidemic because of the poor sanitary apparatus in existence in the public schools where more - likely those children from the underdogs will be seen. Conjunctivitis is one of such communicable disease in western sub - Sahara Africa because of the topography, level of education in the rural communities and the degree of poverty that rocks an average family. Model for transmission dynamics of acute conjunctivitis is proposed and analyzed both analytically and numerically. The model is reformulated as an optimal control problem taking into consideration the effect of proper sanitation and training of the educators; and Maximum Principle was employed to obtain the necessary conditions for existence of optimal control. The basic reproduction number is obtained using the next generation matrix and spectral radius which is less than one when computed. The result shows an agreement of the analytical and numerical solution; in addition, if the sanitation that includes the serenity of the school environment, conduciveness of the classrooms, personal hygiene are dually observed in and outside the school, and education of the caregivers which includes the teachers, menders, parents and even the pupils are articulated properly, the infected pupils shall be decreased drastically over time.
\end{abstract}

Keywords: Conjunctivitis, Stability, Optimal Control, Mathematical Model

\section{Introduction}

Lately mathematical modeling of biological processes, physical process and other epibiological processes have been on the raise, basically because of its ability to incorporate the undermining factors that are so intricate to elucidate. Mathematical biology is a branch of mathematics that has been gaining tremendous interest of scholars recently; modeling of infectious diseases, modeling of growth in the human anatomy, body fluid modeling, and other viable areas. These surges include the solid reputation of such models in laying foundational structures to understanding the complexities of biological processes and the likes. In modeling of infectious diseases, it helps in estimating the threshold parameters, elucidation of the transmission dynamics, in addition to its ability to provide feasible control strategies [1]. Mathematical modeling of infectious diseases has been productive in terms of its pragmatic therapeutic recommendations for treatment and prevention strategies of infectious diseases has been borne, and has been an alternative tool that has been in exploit in the combative approach of infectious diseases [2].

Conjunctivitis is an infectious disease of the eye (s) that is characterized by typically redness or swelling of the white of the eye (Conjunctiva). It is most often caused by virus, bacteria, allergy or chemical irritation (Pollen, smoke, cosmetics or chlorine in water) [3]. The infection is transmitted from an infected person to a susceptible individual through contact with discharge from conjunctivae or upper respiratory tracts of infected persons, contact with contaminated foreign bodies that has been in contact with the eyes. It can also be transmitted vertically to newly born babies due to its direct relation between maternal gonococcal and chlamydial infection. We are interested in conjunctivitis caused by virus rather called Acute Hemorrhagic conjunctivitis (ADC). The incubation period of ADC ranges from $1-3$ days. The symptoms of the infection include tearing, irritation, photophobia, sore throat which usually results in swelling of the lids or purulent discharges [4].

Conjunctivitis infection is commonly contracted in the 
early ages of children by direct or indirect contact with contaminated foreign bodies. It is widely common communicable disease of tropical countries Africa, Asia, Central and South America, and is undoubtable considered as a serious health problem in communities where the appropriate drugs for treatment is scares [5]. The period of infection is about 2 weeks. The infection only affects the eyelids and conjunctiva while part of the black eye is normal, hence it does not affect the sight. Commonly associated with the diseases is its burden that includes loss in contact for hours or days for pupils, loss of savings through medication bills and loss in productivity.

Chowell et al. [6] modeled an outbreak of acute hemorrhagic conjunctivitis by considering a model that was categorized into susceptible, infectious, reported and recovered compartments. The model considered underreporting and behavior changes on the transmission rate of ADC in Mexico. A SEIR model of conjunctivitis was proposed by Unyong and Naowarat [7] that considered a nonlinear incidence on which the local stability was performed; they concluded that an increase in the infected humans was dependent on the decrease in the fraction of the infected individuals. A deterministic mathematical model for the dynamics of conjunctivitis disease that assessed the effect of education campaign on the spread of the diseases was develop by Suratchala et al.[8]. Local stability analysis of mathematical model for hemorrhagic conjunctivitis disease was studied in [9] that they resolved that the endemic and disease free equilibrium are unique and stable. Optimal control model of hemorrhagic conjunctivitis disease was studied using dynamical system that was employed in investigating the potency of the spreading of the epidemic [2]. Their analysis suggests the likelihood of the epidemic to spread in the entire population if the basic reproduction number is exceeded. Even though an outstanding contributions have been achieved by researchers in expatiating viral interactions with human and their biology, the complexities in the life cycle of the virus, highly environmental factors that to a greater extent affects the transmission posed by the virus in drug resistance has thrown a challenge to researchers to come up with combination method in order to eliminate the epidemic.

In this research article, we formulate a dynamical system approach to solving an optimal control model for viral conjunctivitis disease. The purpose of this work is basically to determine the effect of proper sanitation and education of viral conjunctivitis for nursery and primary schools caregivers. The structure of this paper is organized as follows; formulation of model for the viral conjunctivitis in section 2. The optimal control problem that assesses the effect of good sanitation, education on preventive measures and management of the infection since it is self-limited by using time dependent control functions; Pontryagins Maximum Principle is applied to obtain the necessary condition for an optimal solutions. Stability differential equations theory was used to determine both the endemic equilibrium point, basic reproduction number and investigate the stability of the model. Finally, in section 4 the optimal system is numerically solved and computed to investigate the optimum control strategy that would be efficient in curtailing the population in exposed and infected in the compartments.

\section{Formulation of the Transmission Model}

The human population at time $t$ is assumed to be constant because birthrate and death rate of human population are approximately equal. The population is partitioned into four compartments: susceptible human $S(t)$, exposed human $E(t)$, Infected human $I(t)$, and recovered human $R(t)$. The total population at any given time $t$ is

$$
N=S(t)+E(t)+I(t)+R(t) .
$$

The dynamical transmission model for ADC infection is given as follows

$$
\begin{gathered}
\frac{d S}{d t}=\beta N+\theta R-\frac{(1-\pi)}{N} \mu S I-\alpha S \\
\frac{d E}{d t}=\frac{1-\pi}{N} \mu S I-(\kappa+\alpha) E \\
\frac{d I}{d t}=\kappa E-(\rho+\imath+\alpha) I \\
\frac{d R}{d t}=(\rho+\imath) I-(\theta+\alpha) R
\end{gathered}
$$

where

$\beta$ is the birth rate of human population in a school

$\alpha$ is the death rate of human population in a school

$\pi$ is the effectiveness of personal hygiene outside the school

$\kappa$ is the rate that the exposed become infected in a school

$\rho$ is the recovery rate of persons who have seen the doctor

$l$ is the recovery rate of persons who have not consulted the doctor

$\theta$ is the rate of immunity after recovery is lost and the individual become susceptible again

$\mu$ is the probability that virus transmitted from infected pupil to susceptible pupil.

We normalize (2) - (5) by assigning

$$
\hat{S}=\frac{S}{N}, \hat{E}=\frac{E}{N}, \hat{I}=\frac{I}{N}, \hat{R}=\frac{R}{N} .
$$

When (7) is infused into (2) - (5), we obtain

$$
\frac{d \hat{S}}{d t}=\beta+\theta \hat{R}-(1-\pi) \mu \hat{S} \hat{I}-\alpha \hat{S}
$$




$$
\begin{gathered}
\frac{d \hat{E}}{d t}=(1-\pi) \mu \hat{S} \hat{I}-(\kappa+\alpha) \hat{E} \\
\frac{d \hat{I}}{d t}=\kappa \hat{E}-(\rho+\imath+\alpha) \hat{I} \\
\frac{d \hat{R}}{d t}=(\rho+\imath) \hat{I}-(\theta+\alpha) \hat{R} .
\end{gathered}
$$

\subsection{Optimal Control Strategies}

The impact of some control strategies is examined by incorporating them into (2) to (5) which in our work we are considering good sanitation and education of the caregivers in nursery and primary schools. The infection force is reduced by a factor $1-\phi_{1}(t)$, where $\phi_{1}(t)$ represents efforts of maintaining sound sanitation among the individuals. $\phi_{2}(t)$ is introduced as a control variable that represents the education of the caregivers about the infection that includes how to respond when the infection is suspected, proper way of isolation of individuals, follow up with the clinic department and the psychological management. With the introduction of these optimal control strategies which spans better disease management that will save time and resources we can modify (2) - (5) as follows

$$
\begin{gathered}
\frac{d S}{d t}=\beta N+\theta R-\left(1-\phi_{1}(t)\right) \frac{1-\pi}{N} \mu S I-\alpha S \\
\frac{d E}{d t}=\left(1-\phi_{1}(t)\right) \frac{1-\pi}{N} S I-(\kappa+\alpha) E
\end{gathered}
$$

$$
H\left(S, E, I, R, Q, \lambda, \lambda_{2}, \lambda_{3}, \lambda_{4}, t\right)=B_{1} E+B_{2} I+\frac{1}{2}\left(d_{1} \phi_{1}^{2}+d_{2} \phi_{2}^{2}\right)+\lambda_{1} \frac{d S}{d t}+\lambda_{2} \frac{d E}{d t}+\lambda_{3} \frac{d I}{d t}+\lambda_{4} \frac{d R}{d t}
$$

where $\lambda_{i}$ are adjunct variables to be calculated.

Theorem 2.1: There exist an optimal control $\bar{Q}=\left(\phi_{1}^{*}, \phi_{2}^{*}\right) \in Q$ such that $J\left(\phi_{1}^{*}, \phi_{2}^{*}\right)=\min _{\left(\phi_{1}, \phi_{2}\right) \in Q} J\left(\phi_{1}, \phi_{2}\right)$ subject to the control system (11) - (14) with initial conditions at $t=0$.

Theorem 2.2: Given that $\left(S^{*}, E^{*}, I^{*}, R^{*}\right)$ are optimal state solutions and $\left(\phi_{1}^{*}, \phi_{2}^{*}\right)$ are associated optimal control variable for the optimal control problem $(11)-(15)$, then there exists adjoint variables $\lambda_{i}$ which satisfies

$$
\left.\begin{array}{l}
\lambda_{1}^{\prime}=\left(1-\phi_{1}\right)\left(\lambda_{1}-\lambda_{2}\right)(1-\pi) I \frac{(E+I+R)}{N^{2}}+\lambda_{1} \alpha, \\
\lambda_{2}^{\prime}=-B_{1}+\left(\lambda_{2}-\lambda_{3}\right) \kappa+\lambda_{2} \alpha, \\
\lambda_{3}^{\prime}=-B_{2}+\left(1-\phi_{1}\right)\left(\lambda_{1}-\lambda_{2}\right)(1-\pi) I \frac{(E+I+R)}{N^{2}}+\left(\lambda_{3}-\lambda_{4}\right) \rho+\left(\lambda_{3}-\lambda_{4}\right) \phi_{2}+\lambda_{3}(l+\alpha), \\
\lambda_{4}^{\prime}=\left(\lambda_{1}-\lambda_{4}\right) \theta+\lambda_{4} \alpha,
\end{array}\right\}
$$

with the boundary conditions

In [2], the optimal control $\phi_{1}^{*}$ and $\phi_{2}^{*}$ are given as

$$
\lambda_{i}(t)=0 \text { for } i=1,2,3,4 .
$$




$$
\begin{gathered}
\phi_{1}^{*}=\max \left\{\min \left\{\frac{\left(\lambda_{2}-\lambda_{1}\right) \frac{1-\pi}{\beta} \hat{S} \hat{I}}{d_{1} N}, 1\right\}, 0\right\} \\
\phi_{2}^{*}=\max \left\{\min \left\{\frac{\left(\lambda_{4}-\lambda_{3}\right) \hat{I}}{d_{2}}, 1\right\}, 0\right\} .
\end{gathered}
$$

Theorem 2.1 and theorem 2.2 have been proved in [2], we use only the theorem for the optimal solutions. By substituting (20) and (21) into (11) - (14) we obtain

$$
\begin{aligned}
& \frac{d S}{d t}=\beta N+\theta R-\left(1-\max \left\{\min \left\{\frac{\left(\lambda_{2}-\lambda_{1}\right) \frac{1-\pi}{\beta} \mu \hat{S} \hat{I}}{d_{1} N}, 1\right\}, 0\right\}\right) \frac{1-\pi}{\beta N} S I-\alpha S \\
& \frac{d E}{d t}=\left\{1-\max \left\{\min \left\{\frac{\left(\lambda_{2}-\lambda_{1}\right) \mu \frac{1-\pi}{\beta} \hat{S} \hat{I}}{d_{1} N}, 1\right\}, 0\right\}\right) \frac{1-\pi}{\beta N} S I-(\kappa+\alpha) E \\
& \frac{d I}{d t}=\kappa E-\left(\rho+\imath+\alpha+\max \left\{\min \left\{\frac{\left(\lambda_{4}-\lambda_{3}\right) \hat{I}}{d_{2}}, 1\right\}, 0\right\}\right) I \\
& \frac{d R}{d t}=(\rho+\imath) I+\left(\max \left\{\min \left\{\frac{\left(\lambda_{4}-\lambda_{3}\right) \hat{I}}{d_{2}}, 1\right\}, 0\right\}\right) I-(\theta+\alpha) R .
\end{aligned}
$$

\subsection{Analysis of the Dynamic Model}

To obtain the steady state of the model, we equate (7) - (10) to zero. We obtain that

$$
\begin{gathered}
\hat{S}=\frac{\beta(\beta+\theta \hat{R})}{(1-\pi) \hat{I}+\alpha \beta} \\
\hat{E}=\frac{(1-\pi)(\beta+\theta \hat{R})}{(\kappa+\alpha)[(1-\pi) \hat{I}+\alpha \beta]} \hat{I} \\
\hat{I}=\frac{\beta[\kappa \beta(1-\pi)-\alpha(\kappa+\alpha)(\rho+\imath+\alpha)]+\theta \hat{R}}{(\kappa+\alpha)(1-\pi)(\rho+\imath+\alpha)} \\
\hat{R}=\frac{(\rho+\imath)[\kappa \beta(1-\pi)-\alpha(\kappa+\alpha)(\rho+\imath+\alpha)]}{(\rho+\imath) \theta-(\theta+\alpha)(\kappa+\alpha)(1-\pi)(\rho+\imath+\alpha)}
\end{gathered}
$$

$\hat{R}$ can be obtained using (29) or by using the equation $N=\hat{S}+\hat{E}+\hat{I}+\hat{R}$. Therefore we obtain two steady - state;

i When proper sanitation and the community is properly sensitized, the number of persons infected is zero i.e. $\hat{I}=0$, hence we obtain that $\hat{S}=\frac{\beta}{\alpha}, \hat{E}=0, \hat{R}=0$.
Hence $E_{0}(\hat{S}, \hat{E}, \hat{I}, \hat{R})=E_{0}\left(\frac{\beta}{\alpha}, 0,0,0\right)$ is the disease free steady state.

ii The endemic disease steady state is $E_{1}(\hat{S}, \hat{E}, \hat{I}, \hat{R})$, where $\hat{S}, \hat{E}, \hat{I}, \hat{R}$ are as in (26) - (29).

The Basic Reproduction Number

The basic reproduction number $R_{0}$ is the number of secondary cases which one case would produce in a completely susceptible population. We obtain $R_{0}$ by using next generation method and the spectral radius proposed in [10]. We can rewrite (2) - (5) in the system matrix form

$$
\frac{d x}{d t}=F(x)-V(x)
$$

Where $F(x)$ is the rate of new infection in a compartment and $V(x)$ gives the transfer of persons from a compartment to another. Therefore the matrices $F$ and $V$ evaluated at $E_{0}\left(\frac{\beta}{\alpha}, 0,0,0\right)$,

$$
F=\left(\begin{array}{ccc}
0 & 0 & 0 \\
0 & 0 & \frac{\beta \mu(1-\pi)}{\alpha} \\
0 & 0 & 0
\end{array}\right) \text { and } V=\left(\begin{array}{ccc}
\alpha & 0 & \frac{1-\rho}{\alpha} \\
0 & \kappa+\alpha & 0 \\
0 & -\kappa & \rho+l+\alpha
\end{array}\right) \text {. }
$$

The basic reproduction number can be evaluate buy the formula [10].

$$
R_{0}=p\left(F V^{-1}\right)
$$

Where $p$ is the spectral radius of $F V^{-1}$. Therefore

$$
V^{-1}=\frac{1}{(\kappa+\alpha)(\rho+l+\alpha)}\left(\begin{array}{ccc}
\frac{(\kappa+\alpha)(\rho+l+\alpha)}{\alpha} & -\frac{\kappa(1-\pi)}{\alpha^{2}} & -\frac{(1-\pi)(\kappa+\alpha)}{\alpha^{2}} \\
0 & \rho+l+\alpha & 0 \\
0 & \kappa & \frac{(\kappa+\alpha)(1-\pi)}{\alpha}
\end{array}\right)
$$

and

$$
F V^{-1}=\left(\begin{array}{ccc}
0 & 0 & 0 \\
0 & \frac{\beta \kappa(1-\pi) \mu}{\alpha(\kappa+\alpha)(\rho+\imath+\alpha)} & \frac{1-\pi}{\alpha(\rho+\imath+\alpha)} \\
0 & 0 & 0
\end{array}\right) .
$$

Thus,

$$
R_{0}=p\left(F V^{-1}\right)=\frac{\kappa \beta \mu(1-\pi)}{\alpha(\kappa+\alpha)(\rho+\imath+\alpha)} .
$$

Theorem 3.3: The disease free equilibrium is locally asymptotically stable if $R_{0}<1$ and unstable if $R_{0}>1$. 


\subsection{Analysis of the Model}

Generally, the sign of the eigenvalues of a Jacobian matrix $J_{0}$ determines the local stability of each of the steady state of a system of differential equations. The eigenvalues are the solutions of the characteristic equation

$$
\left|J\left(E_{i}\right)-\lambda I\right|=0
$$

where $J\left(E_{i}\right)$ is the Jacobian matrix at a given steady state $E_{i}, i=1,2$ and $I$ is the identity matrix of dimension $3 \times 3$.

i Disease free steady state, $E_{0}\left(\frac{\beta}{\alpha}, 0,0,0\right):$ The Jacobian matrix for the DFE is given as

$$
J_{0}=\left(\begin{array}{ccc}
-\alpha & 0 & -\frac{1-\pi}{\alpha} \\
0 & -(\kappa+\alpha) & \frac{1-\pi}{\alpha} \\
0 & \kappa & -(\rho+l+\alpha)
\end{array}\right)
$$

The characteristic equation is given as

$$
\left|J_{0}-\lambda I\right|=\left|\begin{array}{ccc}
-\alpha-\lambda & 0 & -\frac{1-\pi}{\alpha} \\
0 & -(\kappa+\alpha)-\lambda & \frac{1-\pi}{\alpha} \\
0 & \kappa & -(\rho+\imath+\alpha)-\lambda
\end{array}\right|=0
$$

Therefore

$$
(\lambda+\alpha)\left[\lambda^{2}+(\kappa+2 \alpha+\rho+l) \lambda+(\kappa+\alpha)(\rho+l+\alpha)-\frac{\kappa(1-\pi)}{\alpha}\right]=0 .
$$

From (34) we observe that $\lambda=-\alpha<0$ and to obtain the remaining eigenvalues, we apply the Routh - Hurwitz criteria for stability [11]. From (34) we obtain that

$$
\begin{gathered}
\lambda^{2}+(\kappa+2 \alpha+\rho+\imath) \lambda+(\kappa+\alpha)(\rho+\imath+\alpha)-\frac{\kappa(1-\pi)}{\alpha}=0 . \\
\therefore \lambda^{2}+a_{1} \lambda+a_{2}=0
\end{gathered}
$$

where

$$
\begin{gathered}
a_{1}=\kappa+2 \alpha+\rho+\imath \\
a_{2}=(\kappa+\alpha)(\rho+\imath+\alpha)-\frac{\kappa(1-\pi)}{\alpha} .
\end{gathered}
$$

By Routh - Hurwitz criteria for stability, if $a_{1}>0$ and $a_{2}>0$ then the eigenvalues are negative. Clearly $a_{1}>0$ and we can transform (35) as

$$
a_{2}=(\rho+\imath+\alpha)(\kappa+\alpha)\left(1-R_{0}\right) .
$$

In (36) $a_{1}>0$ when $R_{0}<1$, hence the DFE is asymptotically stable.

ii Endemic State: The Jacobian matrix of the endemic disease steady state is given as

$$
J_{1}=\left(\begin{array}{ccc}
-\alpha-\frac{1-\pi}{\beta} \hat{I} & 0 & -\frac{1-\pi}{\beta} \hat{S} \\
\frac{1-\pi}{\beta} \hat{I} & -(\kappa+\alpha) & \frac{1-\pi}{\beta} \hat{S} \\
0 & \kappa & -(\rho+l+\alpha)
\end{array}\right)
$$

The related eigenvalue problem is given as

$$
\begin{gathered}
\left|J_{1}-\lambda I\right|=\left|\begin{array}{ccc}
-\alpha-\frac{1-\pi}{\beta} \hat{I}-\lambda & 0 & -\frac{1-\pi}{\beta} \hat{S} \\
\frac{1-\pi}{\beta} \hat{I} & -(\kappa+\alpha)-\lambda & \frac{1-\pi}{\beta} \hat{S} \\
0 & \kappa & -(\rho+l+\alpha)-\lambda
\end{array}\right|=0 \\
\therefore \quad \lambda^{3}+r_{1} \lambda^{2}+r_{2} \lambda+r_{3}=0
\end{gathered}
$$

where

$$
\begin{aligned}
& r_{1}=\rho+2 \alpha+\imath+\kappa+\frac{\kappa(1-\pi)}{(\kappa+\alpha)(\rho+l+\alpha)} \\
& r_{2}=\frac{\kappa(1-\pi)}{(\kappa+\alpha)(\rho+l+\alpha)}\left[\left(k+\alpha+\frac{\kappa(1-\pi)}{(\kappa+\alpha)(\rho+l+\alpha)}\right)(\rho+l+\alpha)\right. \\
& \left.\quad+\frac{\kappa(1-\pi)}{(\rho+l+\alpha)}\right]-\kappa(1-\pi) \\
& r_{3}=\left(\frac{\kappa(1-\pi)}{(\kappa+\alpha)(\rho+l+\alpha)}\right)^{2}(\kappa+\alpha)(\rho+l+\alpha)-\alpha \kappa(1-\pi) .
\end{aligned}
$$

We can rewrite (40) in terms of $R_{0}$, to obtain

$$
\left.\begin{array}{l}
r_{1}=\rho+2 \alpha+\imath+\kappa+\alpha R_{0} \\
r_{2}=\alpha R_{0}\left[\left(k+\alpha+\alpha R_{0}\right)(\rho+\imath+\alpha)+\alpha R_{0}(k+\alpha)\right]-k(1-\pi) \\
r_{3}=\left(\alpha R_{0}\right)^{2}(\kappa+\alpha)(\rho+\imath+\alpha)-\alpha \kappa(1-\pi)
\end{array}\right\}
$$

These eigenvalues are negative when the coefficients $r_{1}, r_{2}$ and $r_{3}$ satisfy the Routh - Hurwitz criteria

$$
\begin{aligned}
& \text { i } r_{3}>0 \\
& \text { ii } r_{1}>0 \\
& \text { iii } r_{3} r_{2}-r_{1}>0 \text {. }
\end{aligned}
$$

Clearly $r_{1}>0$, we consider $r_{3}$, which is positive if $\left(\alpha R_{0}\right)^{2}(\kappa+\alpha)(\rho+\imath+\alpha)<\alpha \kappa(1-\pi)$; then for 


$$
\begin{aligned}
r_{3} r_{2}-r_{1}= & {\left[\left(\alpha R_{0}\right)^{2}(\kappa+\alpha)(\rho+\imath+\alpha)-\alpha \kappa(1-\pi)\right] \times } \\
& \alpha R_{0}\left[\left(k+\alpha+\alpha R_{0}\right)(\rho+\imath+\alpha)+\alpha R_{0}(k+\alpha)\right]-k(1-\pi) \\
& -\rho+2 \alpha+\imath+\kappa+\alpha R_{0} .
\end{aligned}
$$

Observe that

$$
\begin{aligned}
& {\left[\left(\alpha R_{0}\right)^{2}(\kappa+\alpha)(\rho+\imath+\alpha)-\alpha \kappa(1-\pi)\right]} \\
& \times\left(\alpha R_{0}\left[\left(k+\alpha+\alpha R_{0}\right)(\rho+\imath+\alpha)+\alpha R_{0}(k+\alpha)\right]-k(1-\pi)\right) \\
& >\rho+2 \alpha+\imath+\kappa+\alpha R_{0} .
\end{aligned}
$$

Hence, $r_{3} r_{2}-r>0$. Then the three condition satisfied the Routh - Hurwitz condition, hence the endemic steady state is locally asymptotically stable.

\section{Numerical Results and Discussion}

The numerical simulation is considered in this section with the intent of obtaining the effect of control strategies on the transmission dynamics of the infection. The state systems of equations are solved using fourth order Runge - Kutta scheme. We study the control effects of proper sanitation and education of the educators; where the proper medication is administered on the spread of the infection. The effects of the control strategies is obtained by comparing numerically the results of the stated work with simulated values taken from [2] and [8] with initial condition $S(0)=1000, E(0)=50, I(0)=$ $10, R(0)=5$, and the parameter values as given in the Table 1 .

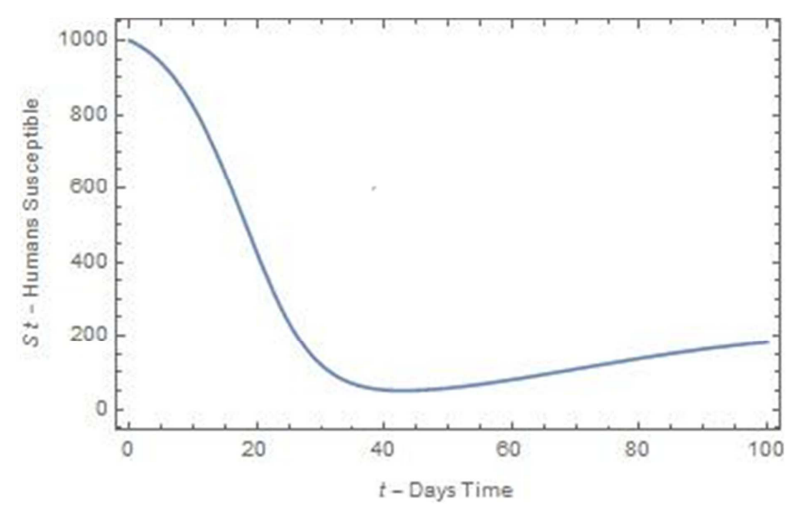

Figure 1. Susceptible Pupils without control.

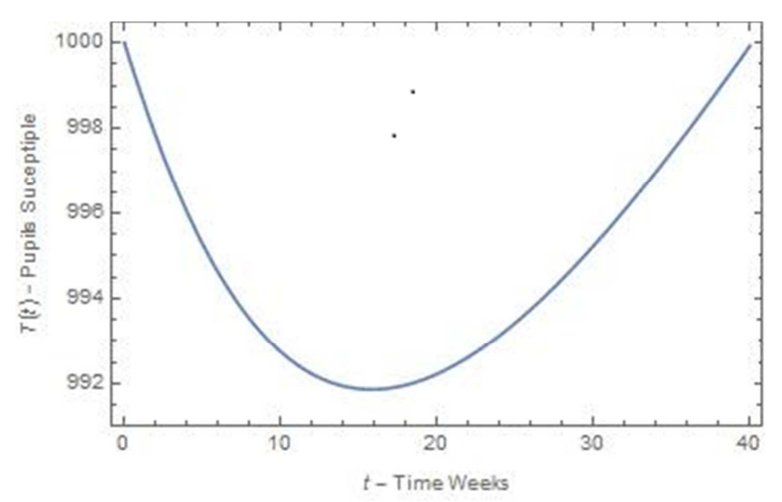

Figure 2. Susceptible Pupils with control.

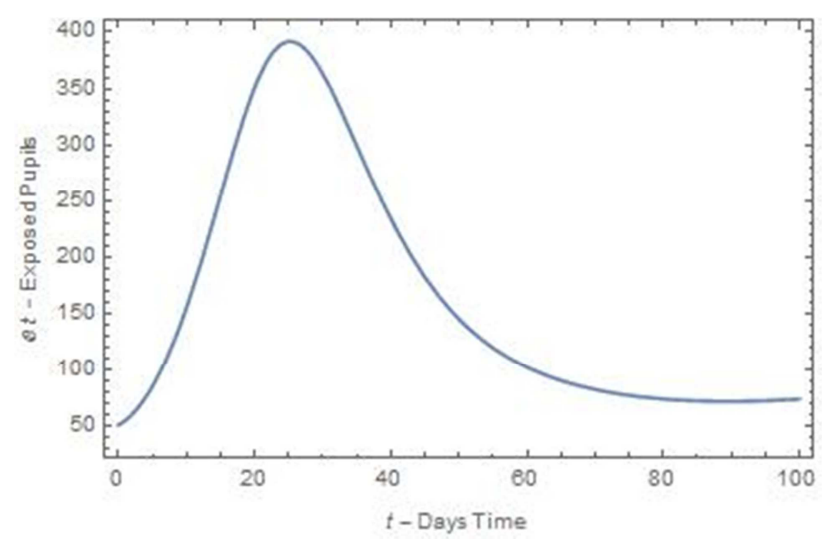

Figure 3. Exposed Pupils without control.

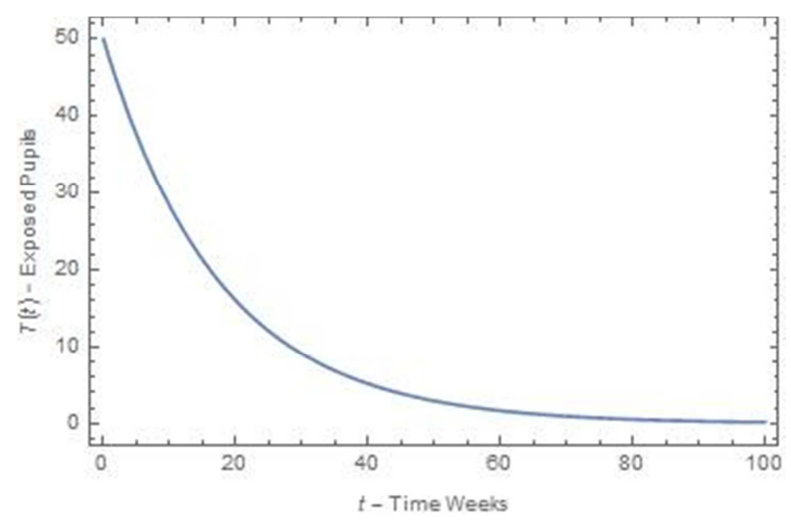

Figure 4. Exposed Pupils without control.

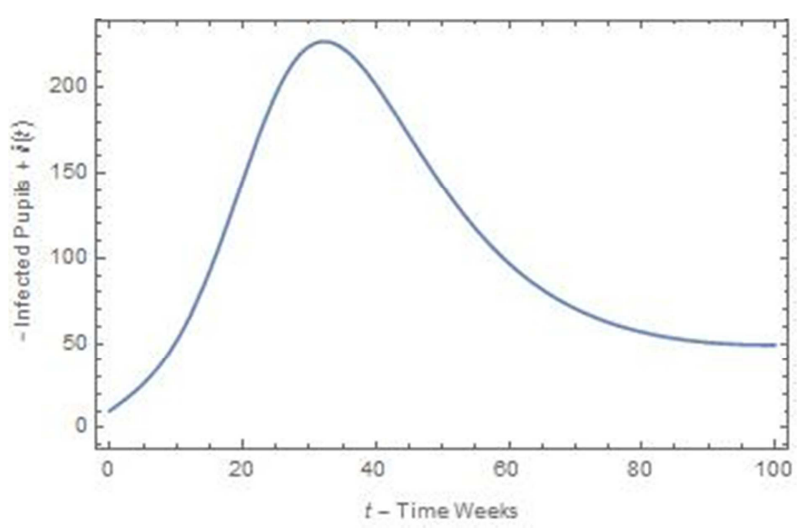

Figure 5. Infected Pupils without control.

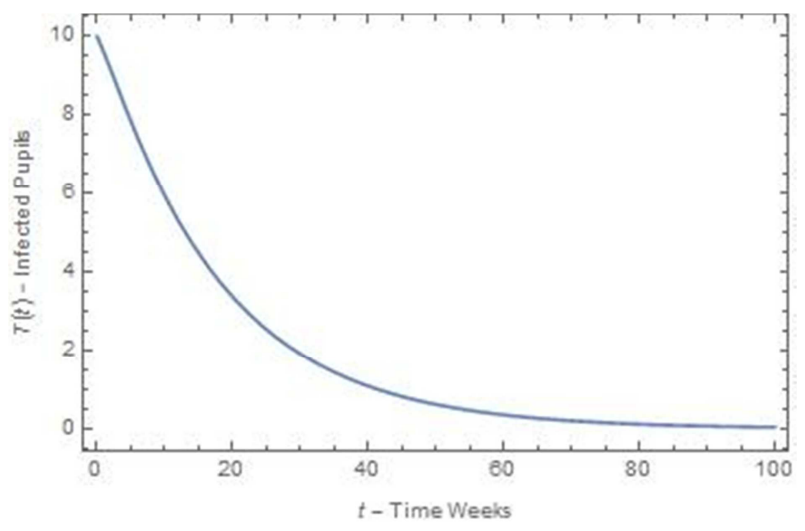

Figure 6. Infected Pupils without control. 


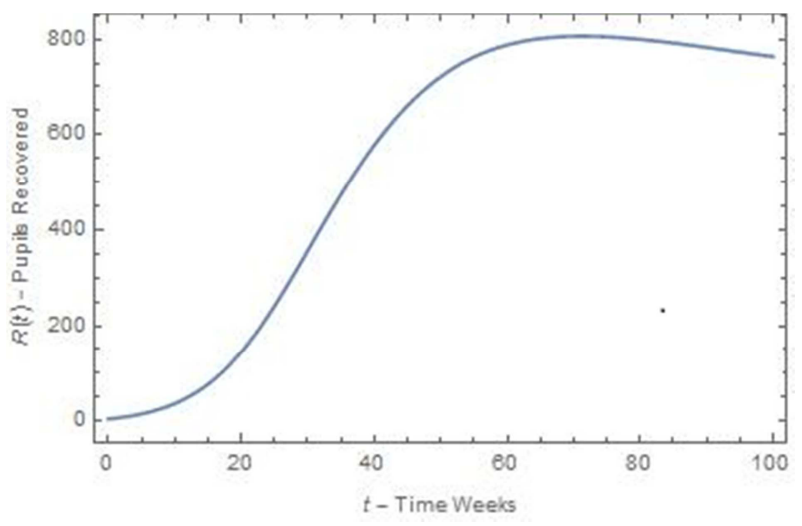

Figure 7. Recovered Pupils without control.

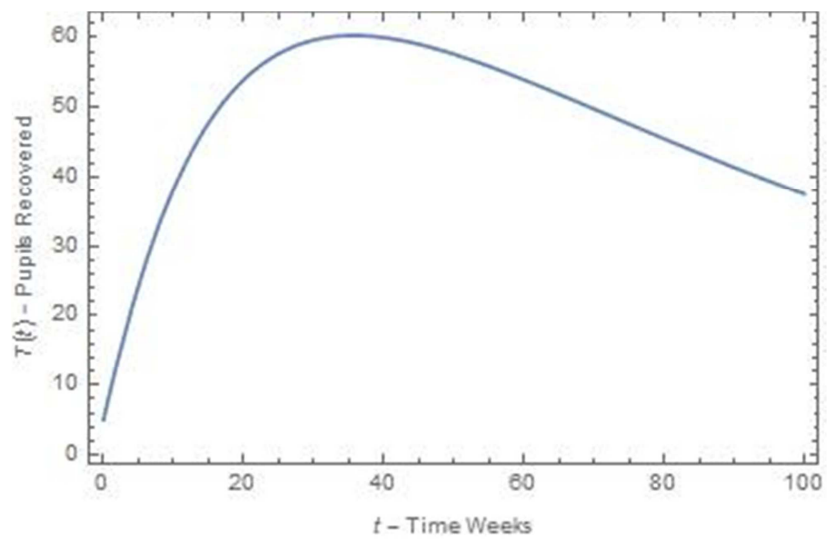

Figure 8. Recovered Pupils without control.

Table 1. Parameters and values used in numerical simulation

\begin{tabular}{llll}
\hline Parameter & Description & Estimated Values & References \\
\hline$\pi$ & effectiveness of personal hygiene outside the school & 0.28 \\
$\beta$ & birth rate of human population in a school & $0.000456 /$ day \\
$\rho$ & recovery rate of persons who have seen the doctor & 0.33 & Assumed \\
$\kappa$ & rate that the exposed become infected in a school & 0.08333 \\
$\theta$ & rate of immunity after recovery is lost and the individual become susceptible again & 0.01 & $0.000456 /$ day \\
$\alpha$ & death rate of human population in a school & 0.004 & {$[8]$} \\
$\mu$ & probability that virus transmitted from infected pupil to susceptible pupil. & 0.005 \\
$l$ & recovery rate of persons who have not consulted the doctor & {$[2]$} & Assumed \\
\hline
\end{tabular}

The values of $\phi_{1}(t)=0.82$ and $\phi_{2}(t)=0.33$ are varied to obtain the optimal solution while other parameters remain unchanged. To examine if it corresponds with the analytic solution, we obtained the eigenvalues and the basic reproduction number: $\lambda_{1}=-0.03254, \lambda_{2}=-0.0002351$, $\lambda_{2}=-0.00004352, R_{0}=0.008539$. These suggest that the endemic equilibrium is locally asymptotically stable since all the eigenvalues are negative and the basic reproduction number is less than one. Figure1 suggest that if proper sanitation is not observe in any public school, over time almost the entire population shall be infected by red eye infection which will have an adverse effect on productivity and learning. Figure 2 shows that if the controls are succinctly administered fewer pupils will leave the susceptible compartment to exposed. Figure 3 and Figure 4 depict that if the controls are not in the system, more pupils will be exposed over time but the reverse is the case when the control is advocated and administered. Figure 5 and Figure 6 shows that when the control is applied that the infected compartment is depopulated. Figure 7 and Figure 8 bring out the beauty of the work; when the control is administered the recovery rate lessens strictly because fewer pupils are being infected by the viral infection.

\section{Conclusion}

Conclusively, two controls functional were introduced to understand their efficacy in preventing the spread of haemorrhagic conjunctivitis disease using mathematical formulated model. In order to attain an optimal control measure, Maximum Principle was used in addition to the analytical and numerical simulation to obtain a feasible control strategy in conjunction with the therapeutic treatment. The study also showed that with the controls administered squarely, that there will be a gross reduction in the number of exposed and infected pupils in any public school. Since the basic reproduction number is less than unity, it suggests that the infection will fizzle out over some time.

\section{References}

[1] Murray J. D., Mathematical Biology I. (2003) Springer Verlag Berlin Heidelbery.

[2] Kyere S. N., Boateng F. A., Hoggar G. F., Jonathan P. (2018) Optimal Control Model of Haemorrhagic conjunctivitis disease. Adv. Comput. Sci, 1(2): 108.

[3] The centers for disease control and prevention, March, 2018.

[4] American Academy of pediatrics. Red Book 2018 - 2021 report of the committee on infectious diseases, $31^{\text {st }}$ Edition.

[5] Medecins Sans Frontiers. Clinical guidelines - Diagnosis and treatment Manuel. 2016 Edition. ISBN 978 -2-37585-001-5.

[6] Chowell G., Shin E., Braver F., Diaz - Duenas P., Hyman J. M. and Castillo - Chavez C. (2005) Modeling the transmission dynamics of acute haemorrhagic conjunctivitis: Application to the 2003 outbreak in Mexico. Stat. Med 25(11): $1840-1857$

[7] Unyong B., Naowarat S., (2014) Stability Analysis of Conjunctivistis model with nonlinear incidence term. Australian Journal of Basic and Applied Sciences. 8(24): $52-$ 58. 
[8] Suratchala S., Anake S., Surapol N. (2015) Effect of education Campaign on Transmission model of conjunctivistis. Australian J. B. and App. Sc. 9(7): $811-815$.

[9] Sireepatch Sangsawang, Tareerat T., Mannissa M., Puntani P. (2012) Local stability Analysis of mathematical model for heamorrhagic conjunctivistis Disease. KMITL Sci. Tech. J. 12(2): $189-197$.

[10] Van den Driessche P., Watmough J. (2002) Reproduction numbers and sub - threshold endemic equilibrium for compartmental models of disease transmission. Math. Biosc. 180: $29-48$.
[11] Robert M. M. (1973) Stability and Complexity in model ecosystem. United States of America. Princeton University press.

[12] Okosun K. O., Rachid O., Marcus N. (2013) Optical Control Strategies and coeffectiveness analysis of a malaria model. Biosys. 111(2): $83-101$.

[13] Lenhart S., Workman J. T. (2007) Optimal control Applied to Biological Models. Chapman and Hall CRC, London. 